\title{
Correlating Removal Rate to Directivity in Copper Chemical Mechanical Planarization
}

\author{
Jeffrey McAllister ${ }^{1,2^{*}}$, Hossein Dadashazar ${ }^{1,2}$, Yasa Sampurno ${ }^{1,2}$, Sung Gyu Kim³, Dongyoul \\ Park $^{3}$, Heeill Kwon ${ }^{3}$, Yongbin Lee ${ }^{3}$, and Ara Philipossian ${ }^{1,2}$ \\ ${ }^{1}$ Araca Inc., Tucson, Arizona, 85718 USA \\ ${ }^{2}$ University of Arizona, Dept. of Chemical and Environmental Engineering, \\ Tucson, Arizona, 85721 USA \\ ${ }^{3}$ Shinhan Diamond, Incheon, 406-840 South Korea
}

The relationship between directivity $(\Delta)$ and removal rate (RR) during copper chemical mechanical planarization (CMP) was investigated. We measured the high-frequency shear and normal forces generated by stick-slip (which has been routinely used to explain micro- and nano-scale interactions that lead to material removal), and found there to be a strong correlation between $\Delta$ (defined as the ratio of variances in shear force to those of normal force) and copper RR so long as the tribological mechanism remained constant. In cases where the tribological mechanism changed from "boundary lubrication" (BL) to "mixed lubrication" (ML), the slope of the straight-line correlation between $\Delta$ and RR was maintained, albeit it was shifted significantly lower. This was due to the ML regime consisting of hydrostatic or buoyant forces supporting the wafer, which led to less variability in frictional forces or less stick-slip events. Additionally, it was found that $\Delta$ and RR increased with sliding velocity while in BL due to an increase in stick-slip 
events. Conversely, $\Delta$ and RR decreased at lower sliding velocities while in ML due to an increase in hydrostatic or buoyant force supporting the wafer.

* Corresponding author: Jeffrey McAllister

* Email: jemcalli@email.arizona.edu

\section{Introduction}

For the past several decades, Chemical mechanical planarization (CMP) has been a key enabler in the scaling of integrated circuit (IC) manufacturing as it is the only means of achieving global and local planarization across the entire wafer surface in an economic manner. During CMP, a rotating polishing pad is placed into direct contact with a rotating wafer. Then, a chemical slurry containing abrasive nanoparticles is injected onto the surface of the pad and transported to the pad-wafer interface through a combination of polisher kinematics and the use of the pores and grooves on the pad surface. When done properly, 3-body contact from the pad surface asperities, the abrasive nanoparticles in the slurry and the features on the surface of the wafer lead to the uniform removal of material from the wafer surface. ${ }^{(1,2)}$ During polishing, the pad surface micro-texture becomes degraded by abrasive wear, plastic deformation on the pad surface, and build-up of polishing byproducts and pad shavings. In order to maintain a steady CMP process, a pad conditioning process is necessary to counteract the degradation of the pad surface micro-texture. ${ }^{(3)}$ During metal CMP the basic, generic, mechanism of material removal has been reported to comprise of two steps. First, a thin passivation layer is formed through a chemical reaction between the slurry and the 
metal surface, and second, this layer is removed through the mechanical abrasion caused by the 3 body contact between pad asperities, slurry nanoparticles and the thin passivation layer. ${ }^{(4,5)}$ It is the repetition of these two steps that creates a dynamic balance between the chemical and mechanical (i.e. passivation layer formation and its subsequent removal) aspects of the CMP process. The balance between the chemical and mechanical aspects of the process is a key factor in determining the quality of the polishing performance (i.e. the tribological, vibrational, thermal and kinetic attributes of the CMP process). ${ }^{(4,5)}$

A necessary series of steps in understanding the kinetics of a CMP process, involves pre-measuring film thicknesses on multiple blanket wafers, polishing them one by one through various combinations of pressures and sliding velocities, cleaning and drying the wafers, and finally determining their post-CMP film thicknesses. These steps are quite straight-forward to perform, but they require sizable manpower as well as time, processing and metrology budgets. In our present study, we attempt to correlate typical wafer-level kinetic data (as noted above) with a certain easily-attainable relationship between data pairs of shear force and normal force (called directivity, or $\Delta$, and defined as the ratio of the variance of shear forces to that of normal forces in a single polishing run) obtained at $1,000 \mathrm{~Hz}$ during processing of the same wafers. $\Delta$, a dimensionless parameter, has its pedigree in the analysis of fine violins. ${ }^{(6-12)}$ How sound radiates from a violin is a key factor in the overall listening experience. The direction of the sound follows the direction of the vibrations in the body of the violin. As the violin is played, both its top plate and bottom plate begin to vibrate. $\Delta$, in this sense, is the ratio of the variance of forces applied to the top plate - which is oriented toward the listener and has two f-holes for the air to exit - to the variance in forces applied to the bottom plate. If the violin's directivity is close to unity, sound emanates in an isotropic and omnidirectional way. If its directivity is higher than unity, the sound 
of the violin travels more toward the listener and further overcomes the sounds of other instruments playing concurrently. For instance, $18^{\text {th }}$ century Italian violins tend to have directivities that range from 1.5 to 2.1 , while very fine modern violins struggle to have their directivities exceed $1.3 .^{(6-12)}$ This anisotropy is analogous to the asymmetry in shear and normal force variances in a typical CMP process. As such, we believe that directivity can be used as a metric for polishing performance. In a CMP process, much like violin playing, as the wafer, the slurry nanoparticles and the pad make physical contact with one another, multiple high-frequency stick-slip events are created that cause vibrations within the wafer-slurry-pad-polisher system. These vibrations are manifested in the form of fluctuations of shear and normal forces during polishing. If these forces can be accurately and precisely measured (not many polishers used today in the industry can do this), then $\Delta$ can be correlated to removal rate (RR). So far, CMP models describing material removal have used average coefficient of friction (COF), but this ignores entirely variance in shear and normal forces. Given the fact that our polisher can measure these forces at a high frequency, we can easily calculate $\Delta$. In fact, our group has recently introduced the parameter into the CMP process. $^{(12-17)}$

Previous work has focused on the vibrations that occur in various CMP processes. ${ }^{(12-21)}$ Fukuroda et al., Hetherington et al., Kojima et al. and Rao et al. studied vibrations in CMP and found that they could be used as an end-point detection technique for polishing different materials. ${ }^{(18-21)}$ However, the authors did not correlate the vibrations to RR. Of the works that have studied vibrations in CMP, several have focused on $\Delta$ 's role in the process. ${ }^{(12-17)}$ Philipossian et al. introduced the concept of $\Delta$ into CMP and found that higher values of $\Delta$ resulted in higher values of copper RR, but the researchers did not analyze enough data points to find a consistent trend. ${ }^{(12)}$ Diaz et al. observed that $\Delta$ was effected by the different polishing regimes that take place in CMP 
and by the design of the specific retaining ring used, but the authors did not perform any wafer polishing to see its effect on RR. ${ }^{(13,14)}$ Peckler et al. also observed that $\Delta$ was affected by a CMP consumable, this time being the pad conditioner. ${ }^{(15)}$ Separately, Peckler et al. found that $\Delta$ somewhat increased with $P \times v$ and found a loose correlation between $\Delta$ and copper RR. ${ }^{(16)}$ Mariscal et al. also found a correlation between $\Delta$ and RR, but for a tungsten CMP application. ${ }^{(17)}$

Mariscal el al. made the following conclusion regarding $\Delta$ based on the fact that material (generally in the form of a chemically softened surface layer) was removed as a result of 3-body contact events among the wafer, slurry nano-particles and pad asperities, and by the relative sliding action of the wafer, in the direction of shearing. The fluctuations in shear force were said to be due to numerous stick-slip events at high frequencies, which combined to strip away the chemically softened surface layer bit by bit. In other words, the removal of material occurred through stickslip events (also described as the variance of shear force). Due to the fact that $\Delta$ is a function of the variance of shear force, it is clear from these works that the relationship between $\Delta$, stick-slip and RR is not yet fully understood. Here, we have performed blanket copper wafer polishing tests and successfully found a very strong correlation between $\Delta$ and RR. In addition, we have described the role that different lubrication regimes play on the correlation between $\Delta$ and RR.

\section{Experimental Apparatus and Procedure}

All tests were performed on an Araca APD-800 polisher and tribometer. ${ }^{(22)}$ The APD-800 is a single-platen research and development polisher and tribometer designed to process substrates measuring up to $300 \mathrm{~mm}$ in diameter. On this tool, the non-oscillating counterclockwise wafer carrier and its drive mechanism sit on a friction table attached to the frame. A load cell connected to the table measures the main component $\mathrm{F}_{\mathrm{y}}$ of the friction force vector. This component runs 
perpendicular to the line connecting the platen and carrier centers. The radial component $F_{x}$ of the friction force is not measured but is known to be small from experiments on a similar $200 \mathrm{~mm}$ tool. ${ }^{(23)}$ The head does not use a contact retaining ring. Instead, a non-contact wafer backing film attached to a water-filled template holds the wafer. The platen and its drive mechanism sit on four load cells that monitor the total force $F_{z}$ transmitted by the wafer to the pad. This includes both the applied load and any fluid forces that develop in the interface. The highest frequency that has been observed in the APD-800 polisher system is less than $500 \mathrm{~Hz} .{ }^{(24)}$ The Nyquist frequency is the maximum frequency that can be computed at a given sampling rate in order to be able to fully process the shear force signal without aliasing problem. ${ }^{(25)}$ As such, all forces in this experiment were measured at $1,000 \mathrm{~Hz}$ because $500 \mathrm{~Hz}$ is the Nyquist frequency of this sampling rate. There is no feedback between the normal force load cells and the carrier. Inside of the carrier, behind the wafer, there is an air chamber that applies the polishing force. An electro-pneumatic transducing regulator controls the chamber pressure. Pressure adjustments can occur but are much slower than the force acquisition frequency. The applied load is periodically checked and calibrated on a static platen. In addition to the signal acquisition hardware, the APD-800 is also equipped with Araca Inc.'s FSX-800 proprietary signal analysis software. These hardware and software features provide the user with the unique ability to acquire accurate real-time shear force and down force measurements. Average shear and normal force, as well as the variance of shear and normal force can then be found from the tens of thousands of instantaneous data points extracted from each wafer run in order to calculate the average COF and $\Delta$ for each wafer run. Additionally, the APD800 also contains a single-point infrared (IR) temperature sensor used for measuring real-time pad surface temperature data during polishing. A more detailed description of the $300-\mathrm{mm}$ wafer polishing system can be found elsewhere. ${ }^{(22)}$ 
In our tests, brand-new $300 \mathrm{~mm}$ blanket electroplated copper wafers were polished at multiple pressures $(P=1.0,1.5$ and 2.0 PSI $)$ and sliding velocities $(v=1.2,1.5$ and $1.8 \mathrm{~m} / \mathrm{s})$ for a total of nine $P \times v$ combinations. Experiments were performed using the Fujimi PL-7106 slurry at a flow rate of $250 \mathrm{~mL} / \mathrm{min}$. This slurry contained less than 1.5 weight $\%$ colloidal silica-based nanoparticles and was pre-mixed by a ratio of 87:10.2:2.8 $\left(\mathrm{H}_{2} \mathrm{O}\right.$ :slurry: $\left.\mathrm{H}_{2} \mathrm{O}_{2}\right)$ as per the manufacture's specification. Prior to polishing, a brand new DowDupont IC-1000 K-grooved polishing pad was broken-in for 60 minutes with the Shinhan CCH212 CVD-coated conditioning disc as shown in Figure 1. The working face of the diamond disc consists of three CVD-coated raised areas shown in black. During break-in, the platen and conditioning disc rotated counterclockwise at 63 and 95 rotations per minute (RPM), respectively. The applied downforce on the conditioner was $5.7 \mathrm{lb}$. Ultra-pure water (UPW) was injected near the pad's center at a flowrate of $250 \mathrm{~mL} / \mathrm{min}$ with the conditioner sweeping across the pad 10 times per minutes. After padbreak in, ten dummy wafers were polished at 1.5 PSI and $1.5 \mathrm{~m} / \mathrm{s}$ in order to ensure a "seasoned", or developed, pad surface. This was ascertained by noting that the coefficient of friction (COF) values after polishing each dummy wafer had become more or less constant. In-situ pad conditioning was next performed followed by polishing tests on the monitor wafers. The pad was rinsed with UPW at $1 \mathrm{~L} / \mathrm{min}$ for 30 seconds between each polish. In order to calculate the RR, the thickness of the copper film was measured with a CMT-SR5000 Sheet Resistance/Resistivity Measurement System before and after polishing, and the difference was divided by the polish time.

\section{Results and Discussion}

Thermal and Tribological Results - Mean values of COF and pad surface temperature are plotted in Figure 2 for all polishing conditions which show that COF and temperature both increase with pressure. This is expected because frictional force (i.e. shear force, or COF) is a function of down 
force and an increase in pressure will cause an increase in force between the pad and wafer. While this trend between pressure, $\mathrm{COF}$ and temperature is straight forward, the trend between sliding velocity, COF and temperature is not, as it depends on the specific value of the pressure. This can be explained by the lubrication results extracted from the Stribeck curve shown in Figure 3 which indicates that the tribological mechanism starts in BL and transitions to ML at higher velocities and lower pressures (i.e. higher values of pseudo-Sommerfeld number). When operating in BL, the wafer, pad and abrasive particles are engaged in intimate contact, such that it is unlikely that a hydrostatic or buoyant force exists due to the fluid supporting the wafer. ${ }^{(26,27)}$ In this lubrication regime, COF exhibits little change with pseudo-Sommerfeld number. This translates to the very small change in COF with the sliding velocity as observed in Figure 2 at the highest pressure. Although, while the COF is not changing with sliding velocity at the highest pressure, temperature is increasing. This is likely due to the ability of the K-groove shapes machined on the pad surface to retain the slurry. ${ }^{(27)}$ At higher values of sliding velocity, fresh slurry introduced on to the pad surface is not able to dilute the retained slurry and lower the temperature of the pad surface as effectively as it does at lower values of sliding velocity. In comparison, at lower values of pressure polishing performance transitions to ML. In this regime, the wafer and the pad are not in intimate contact with one another. Some abrasives may be in direct contact with the pad and wafer, but much less than in the case of BL. A fluid film layer will develop partially separating the wafer and the pad, and a hydrostatic or buoyant force will then exist due to this fluid supporting the wafer. $^{(26,27)}$ It can now been seen from Figure 2 that $\mathrm{COF}$ is decreasing with sliding velocity in this regime while temperature is changing to a lesser extent. This makes sense since COF decreases in ML, therefore causing less heat due to friction to dissipate towards and into the pad. The increase in pad temperature due to slurry retention is then offset by the decrease in COF. 
Force cluster plots are shown in Figure 4. Each cluster is made of 60,000 data points and the reported COF values are the average of the ratios of all 60,000 shear and normal force data pairs. A description of the various cluster shapes is noteworthy here: At the lowest pressure, variations in shear force are much smaller due to the fact that these polishing conditions fall solely in the ML regime. At higher pressures, clusters seem to have distinct core regions (where the concentration of individual data points is the greatest) surrounded by halo regions (where the concentration of data points become progressively sparse as the data pairs move away from the core's center). As pressure is increased, the clusters seem to become stretched in the longitudinal direction. In many cases, this stretching is asymmetrical where the halo is expanded in only one direction (towards the right-hand side) and is no longer encompassing the core. In a recent study, Borucki et al. analyzed similar force clusters obtained from polishing blanket copper wafers and discovered the existence of certain structures in the halo regions. ${ }^{(28)}$ On one side of the clusters analyzed, a high shear force was paired with a low normal force while on the opposite side, a low shear force was paired with a high normal force (the same is more or less true in our case). Borucki et al. attributed the structures to the development of fluid dynamic forces in the pad-slurry-wafer interface which caused repetitive high-frequency changes in the attitude (i.e. altitude, pitch and bank) of a wafer being polished. ${ }^{(28)}$ Since both solid contact and fluid pressures contributed to normal force, while shear forces were solely governed by solid contact pressure, the authors deduced that slurry availability was one of the factors dictating the force cluster shapes in his work. In our case, we believe that the myriad shapes shown in Figure 4 are also caused by the periodic development of high-frequency fluid pressure and suction events in the wafer-slurry-pad interface, which are affected by the polishing regime. When polishing is in the BL regime, these fluid pressure and suction events have the ability to affect variations in shear force more greatly since the wafer- 
slurry-pad interface is so intimate. As the polishing regime transitions to ML, these fluid pressure and suction events have less of an effect on shear force variations since hydrostatic or buoyant forces are now beginning to support the wafer. When the pressure is decreased to the lowest value used in this study (1.0 PSI), these fluid pressure and suction events are either less pronounced or have less of an effect on shear force variations due to the wafer now being greatly supported by hydrostatic or buoyant forces.

While these qualitative observations in the force cluster plots can be explained, such visual observations are not thorough or scientific enough; the variations of forces need to be compared in a more quantitative way. This can be done by analyzing the parameter $\Delta$ (again, defined as the ratio of the variance of shear forces to that of normal forces for all 60,000 data pairs in a single polishing run). The importance of $\Delta$ can be explained theoretically. Generally speaking, in CMP, the 3-body contact between abrasive NPs, pad asperities and the wafer surface can result in NPs rolling, or sticking and slipping on the wafer surface. ${ }^{(29)}$ This stick and slip results in the generation of mechanical forces which can be measured through $\Delta$. When studying the tribology of CMP, stick-slip refers to cyclic fluctuations in the magnitudes of frictional force and relative velocity between the wafer and the pad. It is usually associated with a relaxation oscillation that depends on a decrease of the COF with increasing sliding velocity. True stick-slip, in which each cycle consists of a stage of actual stick followed by a stage of overshoot (i.e. slip) requires that the kinetic COF (i.e. the COF parameter being measured in this study) is lower than the static COF (i.e. corresponding to the maximum friction force that must be overcome to initiate macroscopic motion between the wafer and pad). ${ }^{(30)}$ It is precisely due to the fact that we cannot measure static COF, that we have resorted to reporting the mean dynamic $\mathrm{COF}$ (which is the average of the ratios of all 60,000 shear and normal force data pairs) in our Stribeck curves. This however does not 
completely capture the differences in the tribological attributes of the process. Simply put, mean COF does not consider the nuances associated with stick-slip events, nor does it reflect any of the instantaneous changes in wafer attitude (i.e. altitude, pitch and bank). Indeed, as shown in Figure 3, there is a change in mean $\mathrm{COF}$ as a function of pseudo-Sommerfeld number, however, a measure of the variance of shear force to the variance of normal force reflects the extent of stick-slip events which dictates the actual shape of the data clusters in each case. Instead of measuring the ratio of the average forces generated during this stick-slip process, $\Delta$ measures the ratio of their variances and is therefore able to quantify these stick-slip events with higher values of $\Delta$ representing higher frequencies of stick-slip events.

Similar to the Stribeck curve in Figure 3, $\Delta$ is plotted against pseudo-Sommerfeld number in Figure 5. By comparing these two plots, a trend can be observed based on the lubrication regimes involved. From the Stribeck curve in Figure 3, when polishing is at low values of pseudoSommerfeld number the processes is in BL where changes in sliding velocity result in small changes in COF. While in this regime, $\Delta$ increases with pseudo-Sommerfeld number in Figure 5 due to increases in velocity resulting in more stick-slip events. From Figure 3, as pseudoSommerfeld number is increased, polishing transitions to ML. At this point $\Delta$ reaches a maximum in Figure 5 and then begins to decrease with the pseudo-Sommerfeld number. In the ML regime $\Delta$ is now dominated by changes in pressure, indicating that at lower pressures, hydrostatic or buoyant forces supporting the wafer lead to less variability of frictional forces or less stick-slip events. This can be backed up further by the plot of $\Delta$ vs $P \times v$ in Figure 6. From this plot it can be seen that sliding velocity has little effect on $\Delta$ until the highest value of pressure is reached (i.e. when the polishing process is in BL). This makes sense due to the fact that the solid contact and fluid forces taking place in each regime are different resulting in differing amounts of stick-slip. 
Kinetic Results - In keeping with the trends observed from the thermal and tribological results, Figure 7 demonstrates that copper RR is also increasing with $P \times v$. This is expected since we've shown that $\mathrm{COF}$ and temperature also increase with increasing with $P \times v$. Additionally, it appears that, like $\Delta$, RR does not change much with sliding velocity until polishing is performed at the highest pressure. When it comes to RR, it is best to express things graphically not as a function of $P \times v$, but rather as a function of $C O F \times P \times v$ simply due to the fact that the latter relationship will obviate any departures from Prestonian behavior (Figure 8). Results show that, regardless of the lubrication regime, similar trends in RR versus $C O F \times P \times v$ are observed in that as the product of COF and mechanical power increases, so does copper RR. However, depending on the pressure, or, in turn, the polishing regime, RR behaves differently with changes in sliding velocity. At lower pressures (i.e. ML) RR decreases with increasing sliding velocity, while at higher pressures (i.e. BL) RR increases with sliding velocity. This makes sense because the removal of material occurs through stick slip-events. In BL, increasing sliding velocity increases these stick-slip events and therefore increases RR. However, in ML, increasing sliding velocity increases the hydrostatic or buoyant forces supporting the wafer and therefore decreases stick-slip events and the RR. Since these stick-slip events cause the removal of material in CMP, and our tool is capable of measuring the variations in forces caused by such events, there should be a strong correlation between RR and $\Delta$ (Figure 9), which indeed turns out to be the case. The reasoning behind the two separate yet identically-sloped lines is again due to the change in lubrication regime. Following the same trend as described earlier, $\Delta$ increases with RR while in BL and $\Delta$ decreases with RR while in ML. However, regardless of the regime that the polishing conditions follow, the trend between $\Delta$ increases with RR is the same (as shown by the identical slopes of the fitted lines). This indicates 
that the measured variations in forces which are captured by the parameter, $\Delta$, are the direct result of material removal on the wafer surface. While COF is not able to capture this fully, $\Delta$ is, since it represents the extent of stick-slip events that are occurring in a particular CMP process. It should be noted that our entire set of experiments was repeated (keeping all conditions exactly the same) with another CVD-coated Shinhan conditioner disc that had a different design and performance characteristics (Shinhan CCH23), and nearly identical results (in terms of the relationship between $\mathrm{RR}$ and $\Delta$, and the role of the lubrication regime) were achieved. The complete set of results are not shown here for the sake of brevity, however, the plot of RR vs $\Delta$ is shown in Figure 10 . The data in Figure 10 provides further credibility to our general approach and way of thinking presented here as the same set of conclusions can be made.

\section{Conclusion}

We investigated the correlation between $\Delta$ and copper RR. A necessary series of steps in determining the RR of a CMP process, involves pre-measuring film thicknesses on multiple blanket wafers, polishing them one by, cleaning and drying the wafers, and finally determining their post-CMP film thicknesses. These steps are quite straight-forward to perform, but they require sizable manpower as well as time, processing and metrology budgets. Since stick-slip causes RR in CMP, and directivity is able to measure the high frequency forces generated by stickslip, a strong correlation between $\Delta$ and copper RR was found. It was also determined that this relationship between $\Delta$ and copper RR did not depend on the polishing regime that was occurring during the process. Regardless of polishing regime the relationship correlation between $\Delta$ and copper RR was identical, however, the values of $\Delta$ that occurred were much lower in the ML 
regime. This was due to the ML regime consisting of hydrostatic or buoyant forces supporting the wafer, which led to less variability of frictional forces (i.e. less stick-slip events). Additionally, it was found that $\Delta$ and RR increased with sliding velocity while in the BL regime, due to an increase in stick-slip events, and $\Delta$ and RR decreased with sliding velocity while in ML, due to an increase in hydrostatic or buoyant forces which supported the wafer. This new understanding of the relationship between $\Delta$ and $R R$, and how it depends on the lubrication regime, provides a new way to estimate the polishing behavior in a much less labor-intensive way as compared to the measurement of pre- and post-polish film thicknesses. 


\section{References}

1. J. Steigerwald, S. Murarka and R. Gutmann. Chemical Mechanical Planarization of Microelectronic Materials. John Wiley \& Sons, 2008.

2. P. Zantye, A. Kumar and A. Sikder, Materials Science and Engineering: R: Reports, 45, 89 (2004).

3. Y. Li. Microelectronic Applications of Chemical Mechanical Planarization. John Wiley \& Sons, 2007.

4. F. Kaufman, D. Thompson, R. Broadie, M. Jaso, W. Guthrie, D. Pearson and M. Small, J. Electro. Soc., 138, 11 (1991).

5. R. Singh, S. Lee, K. Choi, G. Basim, W. Choi, Z. Chen and B. Moudgil, MRS Bulletin, 752-760 (2002).

6. J. Meyer, J. Acoust. Soc. Am., 51, 1994-2009 (1972).

7. H. Dünnwald, Catgut Acoust. Soc. J., 1-5 (1991).

8. L. Wang, C. Burroughs, Catgut Acoust. Soc. J., 3, 7-15. (1999).

9. G. Bissinger, D. Oliver, Sound Vibrations, 41, 10-15 (2007).

10. N. Harris and F. Fahy, J. Violin Soc. Am., 211-223 (2009).

11. J. Curtin and T. Rossing, The Science of String Instruments, Springer, 209-244 (2010).

12. A. Philipossian, Y. Sampurno and L. Peckler, Micromachines, 9, 37 (2018).

13. G. Diaz, Y. Sampurno and A. Philipossian, ECS J. Solid State Sci. Tehcnol., 7, 9 (2018).

14. G. Diaz, Y. Sampurno, S. Theng and A. Philipossian, ECS J. Solid State Sci. Tehcnol., 8, $5(2019)$.

15. L. Peckler, Y. Mu, C. Stuffle, Y. Sampurno and A. Philipossian, ECS J. Solid State Sci. Tehcnol., 7, 5 (2018). 
16. L. Peckler, R. Han, Y. Sampurno and A. Philipossian, ECS J. Solid State Sci. Tehcnol., 7, 3 (2018).

17. J. Mariscal, J. McAllister, Y. Sampurno, J, Sierra Suarez, L. Borucki and A. Philipossian, ECS J. Solid State Sci. Tehcnol., 8, 5 (2019).

18. A. Fukuroda, K. Nakamura and Y. Arimoto: In situ CMP monitoring technique for multilayer interconnection. In: IEDM Proceedings, Washington, DC USA (1995).

19. D. Hetherington, D. Stein, J. Lauffer, E. Wyckoff and D. Shingledecker: Analysis of insitu vibration monitoring for end-point detection of CMP planarization processes. In: SPIE 3743 Proceedings, Edinburgh, United Kingdom (1999).

20. T. Kojima, M. Miyajima, F. Akaboshi, T. Yogo, S. Ishimoto and A. Okuda, IEEE Transactions on Semiconductor Manufacturing, 12, 3 (2000).

21. P. Rao, M. Bhushan, S. Bukkapatnam, Z. Kong, S. Byalal, O. Beyca, A. Fields and R. Komanduri, IEEE Transactions on Semiconductor Manufacturing, 27, 1 (2014).

22. http://aracainc.com/equipment/r-and-d-polishers/

23. Y. Sampurno, Ph.D. dissertation, University of Arizona, Tucson, AZ (2008).

24. Y. Sampurno, F. Sudargho, Y. Zhuang, M. Goldstein and A. Philipossian, Thin Solid Films, 516 (2008).

25. R. Bracewell, The Fourier Transform and its Applications., McGraw-Hill, 1994.

26. Y. Moon, Ph.D. dissertation, University of California, Berkeley, CA (1999).

27. A. Philipossian and S. Olsen, Jap. J. of Applied Physics, 42, 10 (2003).

28. L. Borucki, Y. Sampurno and A. Philipossian, Why Did the Wafer Slip? Deduction of Wafer Attitude Data from Force Data. In: ICPT CMP Technology Proceedings, Seoul, Republic of Korea (2018). 
29. F. Ilie and G. Ipate, Lubricants., 5, 15 (2017).

30. D. DeNardis, J. Sorooshian, M. Habiro, C. Rogers and A. Philipossian, Jap. J. of Applied Physics, 42, 1 (2003). 


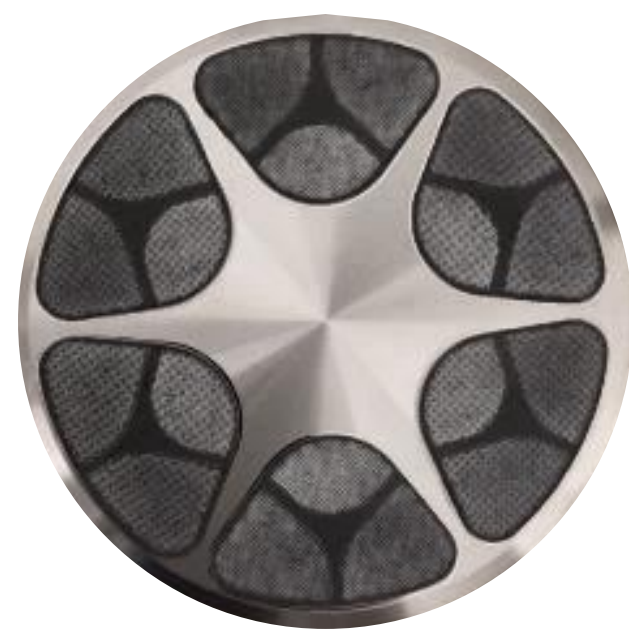

Figure 1: Disc design for the CCH212 pad conditioner. 

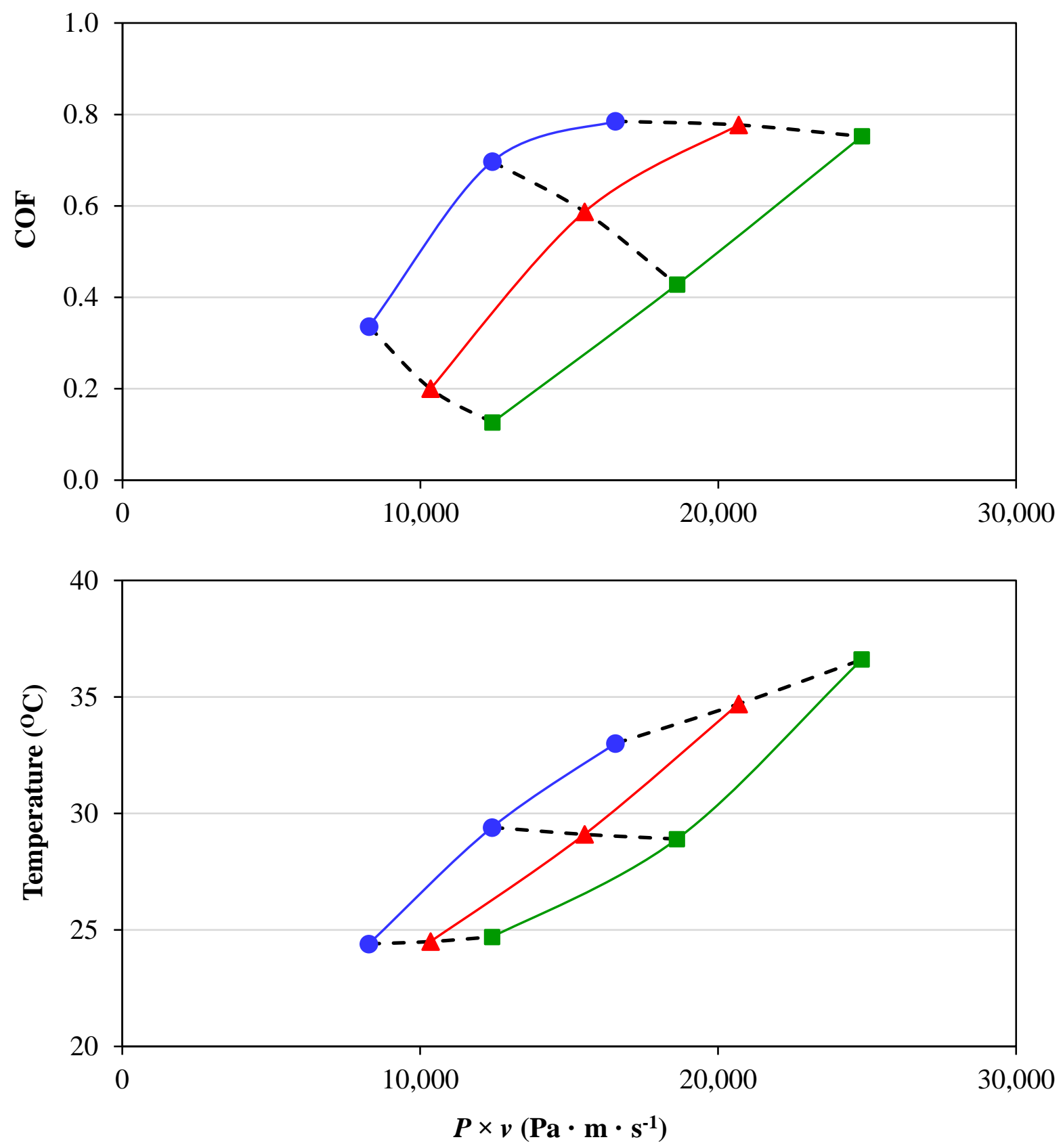

Figure 2: COF and Temperature vs $P \times v$ at sliding velocities of 1.2 (blue circles), 1.5 (red triangles) and 1.8 (green squares) $\mathrm{m} / \mathrm{s}$ - dashed lines represent isobars. 


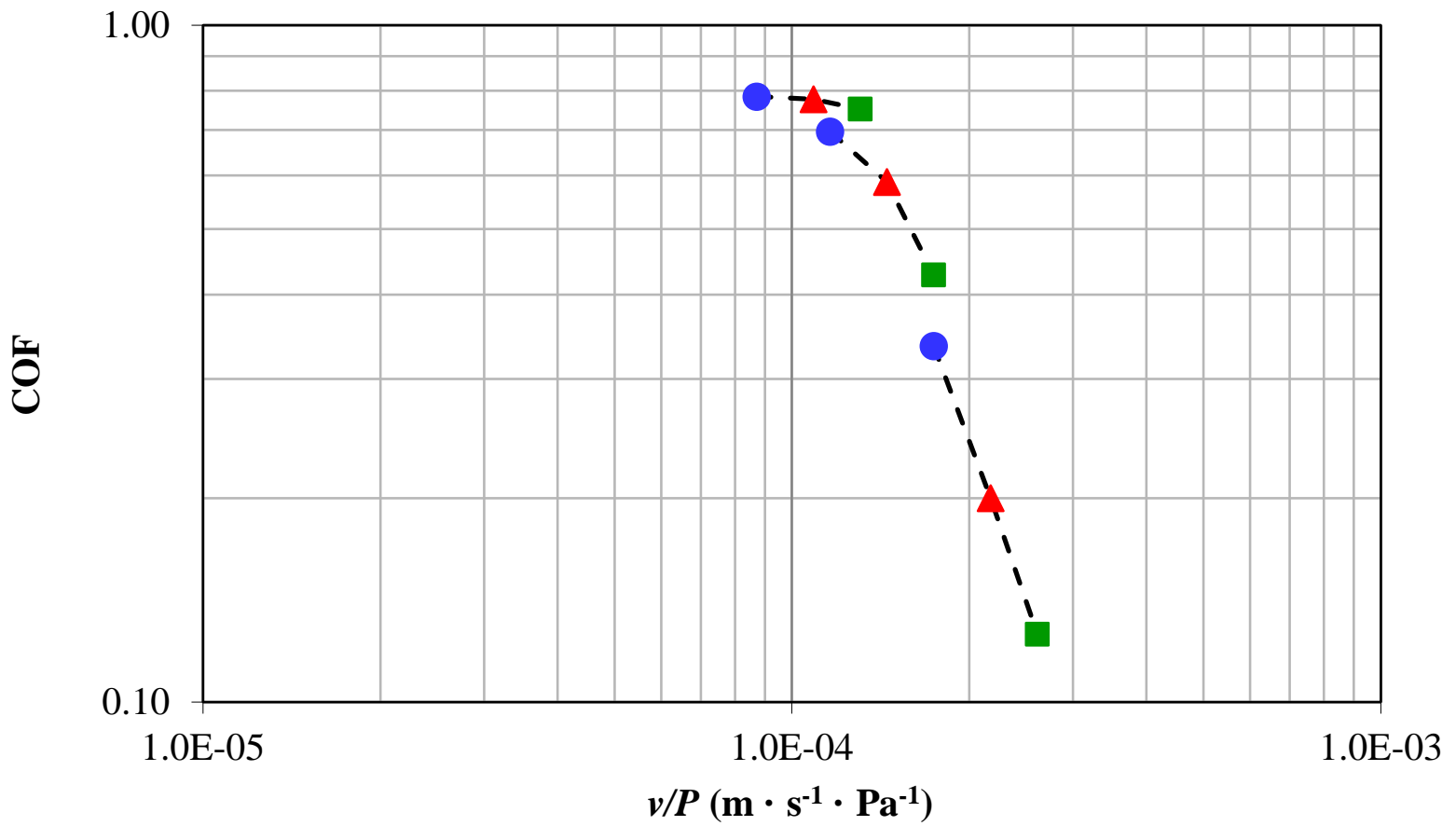

Figure 3: Stribeck Curve at sliding velocities of 1.2 (blue circles), 1.5 (red triangles) and 1.8 (green squares) $\mathrm{m} / \mathrm{s}$ - dashed lines represent isobars. 

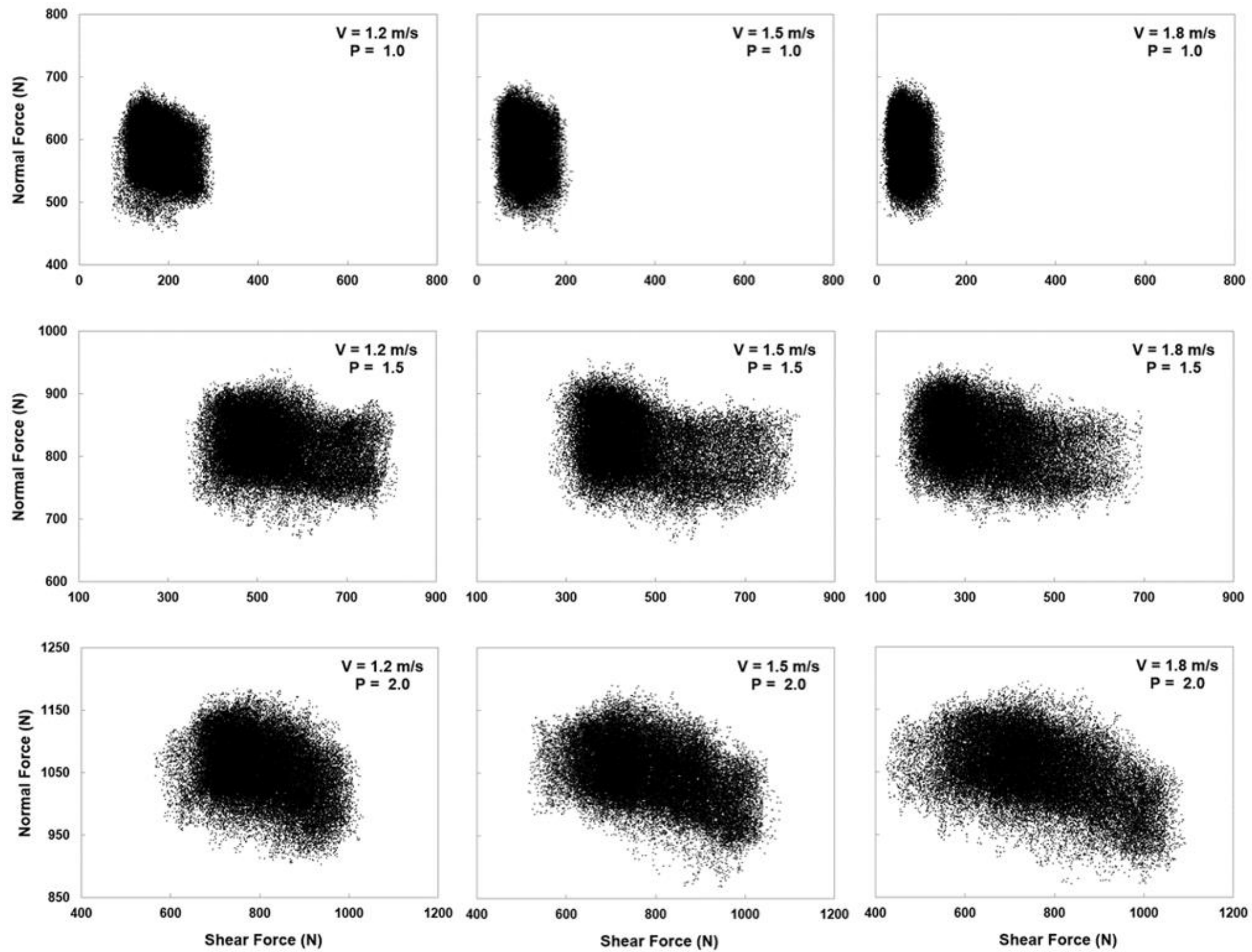

Figure 4: Cluster plots of shear force vs. normal force for all 9 combinations of $P \times v$. 


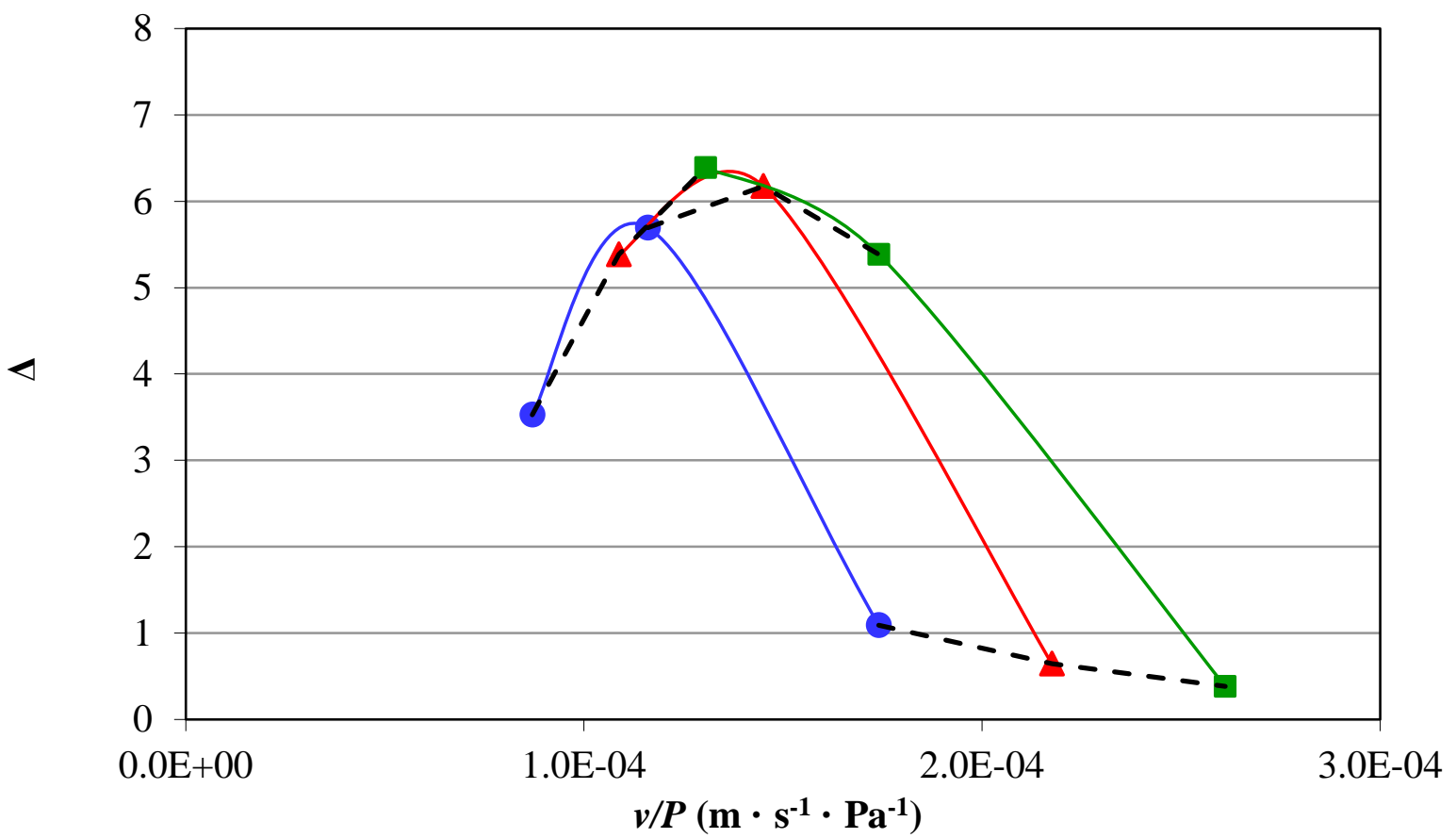

Figure 5: $\Delta$ vs pseudo-Sommerfeld number at sliding velocities of 1.2 (blue circles), 1.5 (red triangles) and 1.8 (green squares) $\mathrm{m} / \mathrm{s}$ - dashed lines represent isobars. 


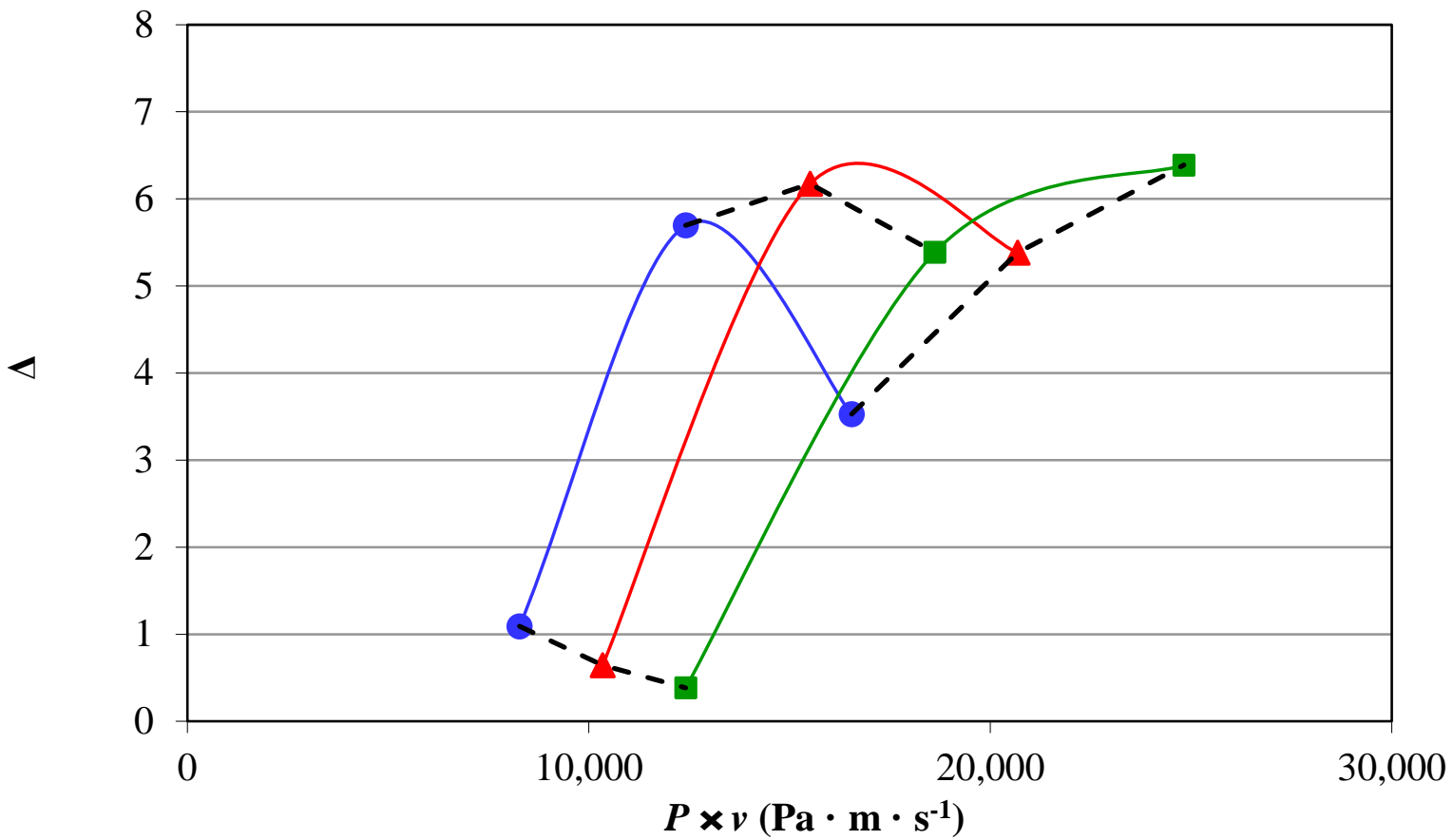

Figure 6: $\Delta$ vs $P \times v$ at sliding velocities of 1.2 (blue circles), 1.5 (red triangles) and 1.8 (green squares) $\mathrm{m} / \mathrm{s}$ - dashed lines represent isobars. 


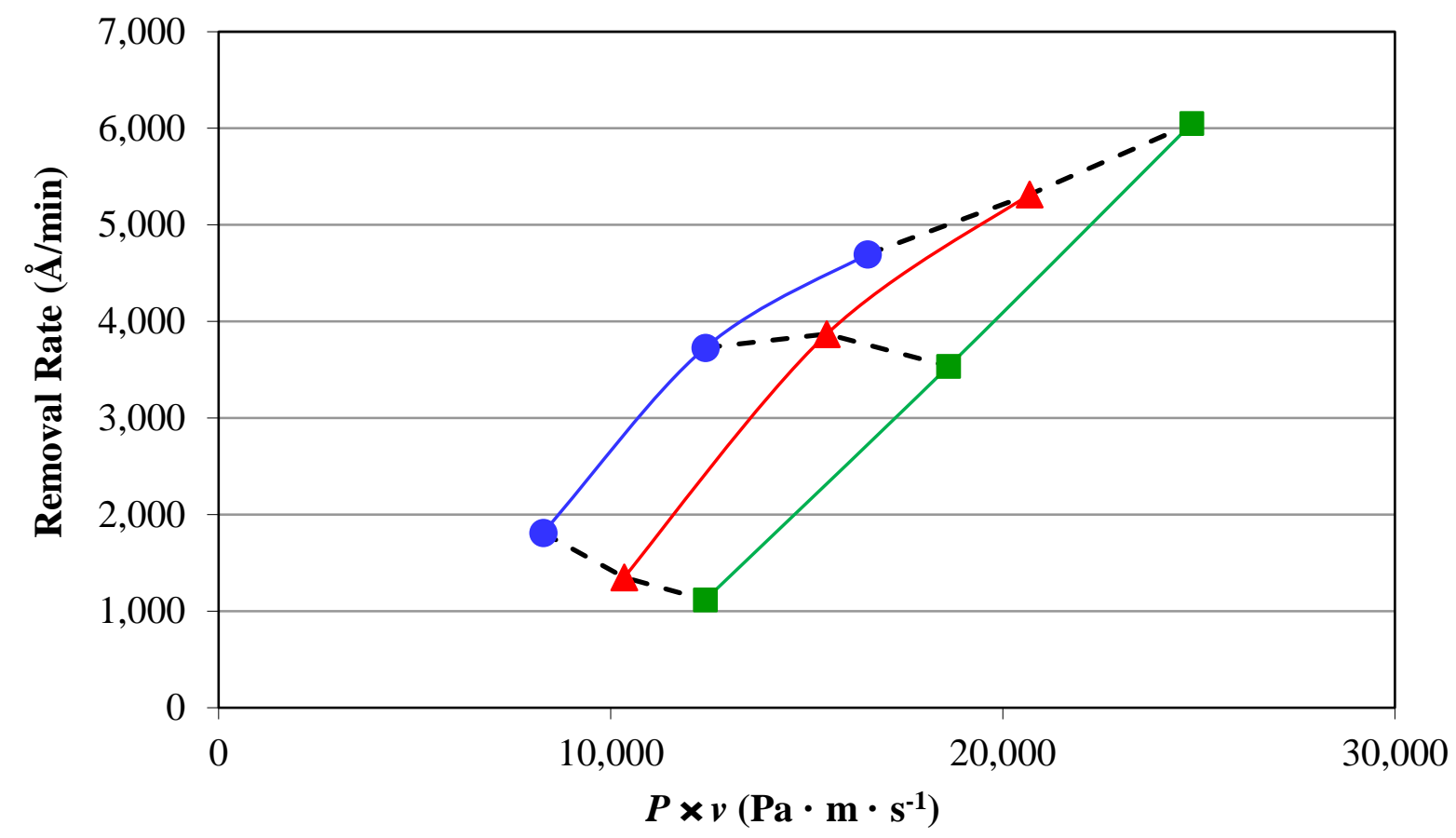

Figure 7: Copper Removal Rate vs $P \times v$ at sliding velocities of 1.2 (blue circles), 1.5 (red triangles) and 1.8 (green squares) $\mathrm{m} / \mathrm{s}$ - dashed lines represent isobars. 


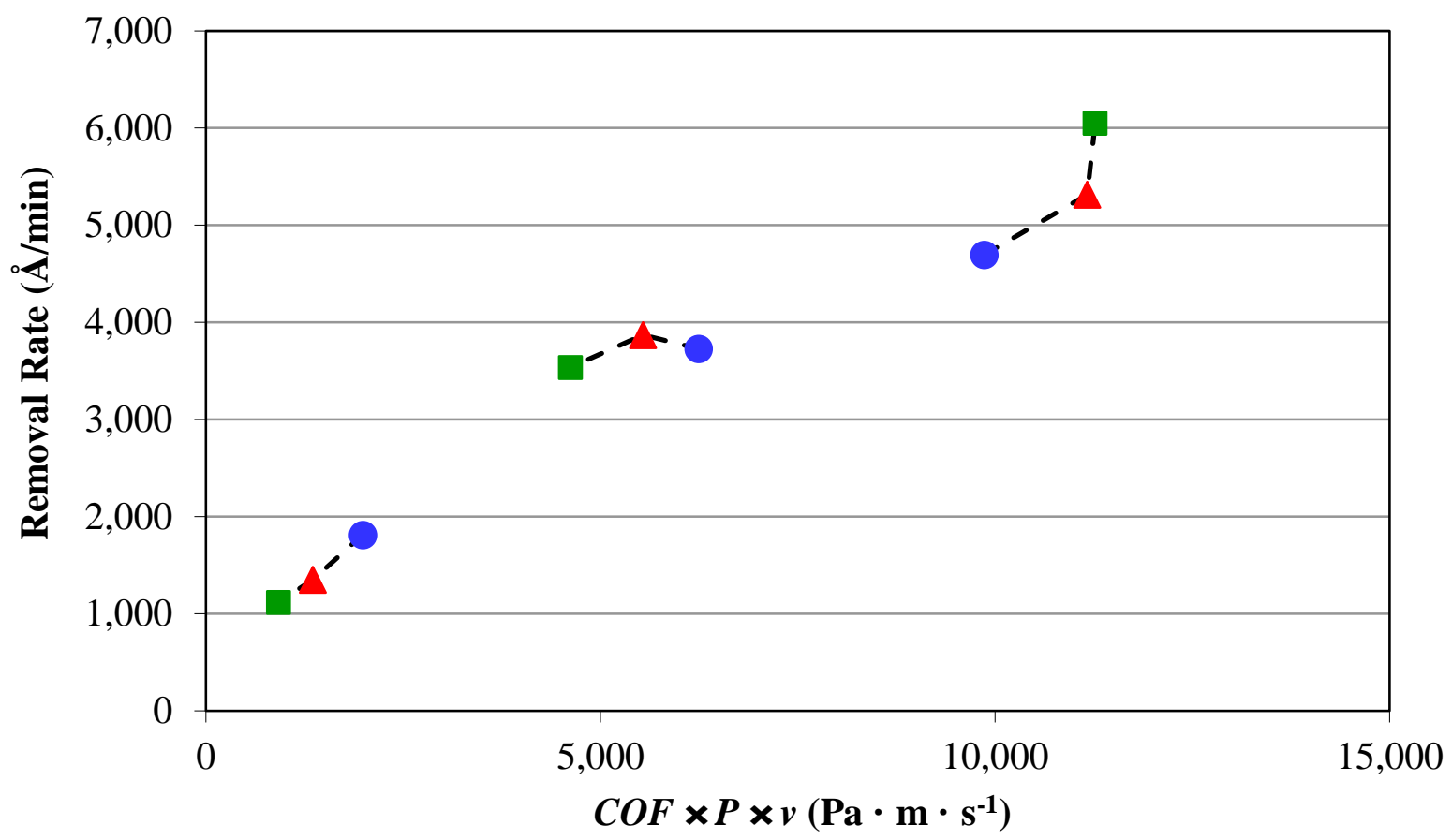

Figure 8: Copper Removal Rate vs COF $\times P \times v$ at sliding velocities of 1.2 (blue circles), 1.5 (red triangles) and 1.8 (green squares) $\mathrm{m} / \mathrm{s}$ - dashed lines represent isobars. 


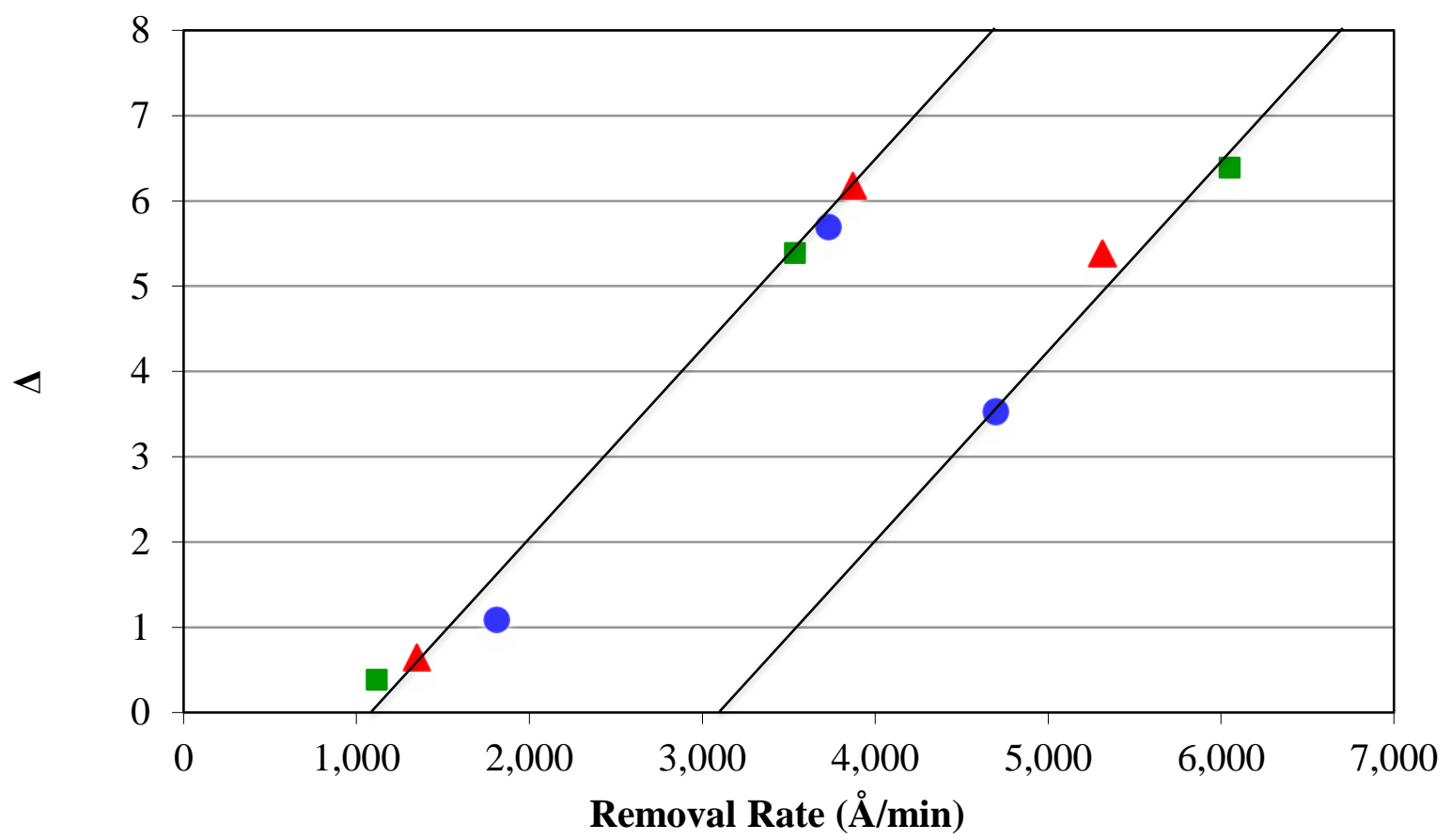

Figure 9: Copper Removal Rate vs $\Delta$ at sliding velocities of 1.2 (blue circles), 1.5 (red triangles) and 1.8 (green squares) $\mathrm{m} / \mathrm{s}$ - Using the Shinhan CCH212 conditioner. 


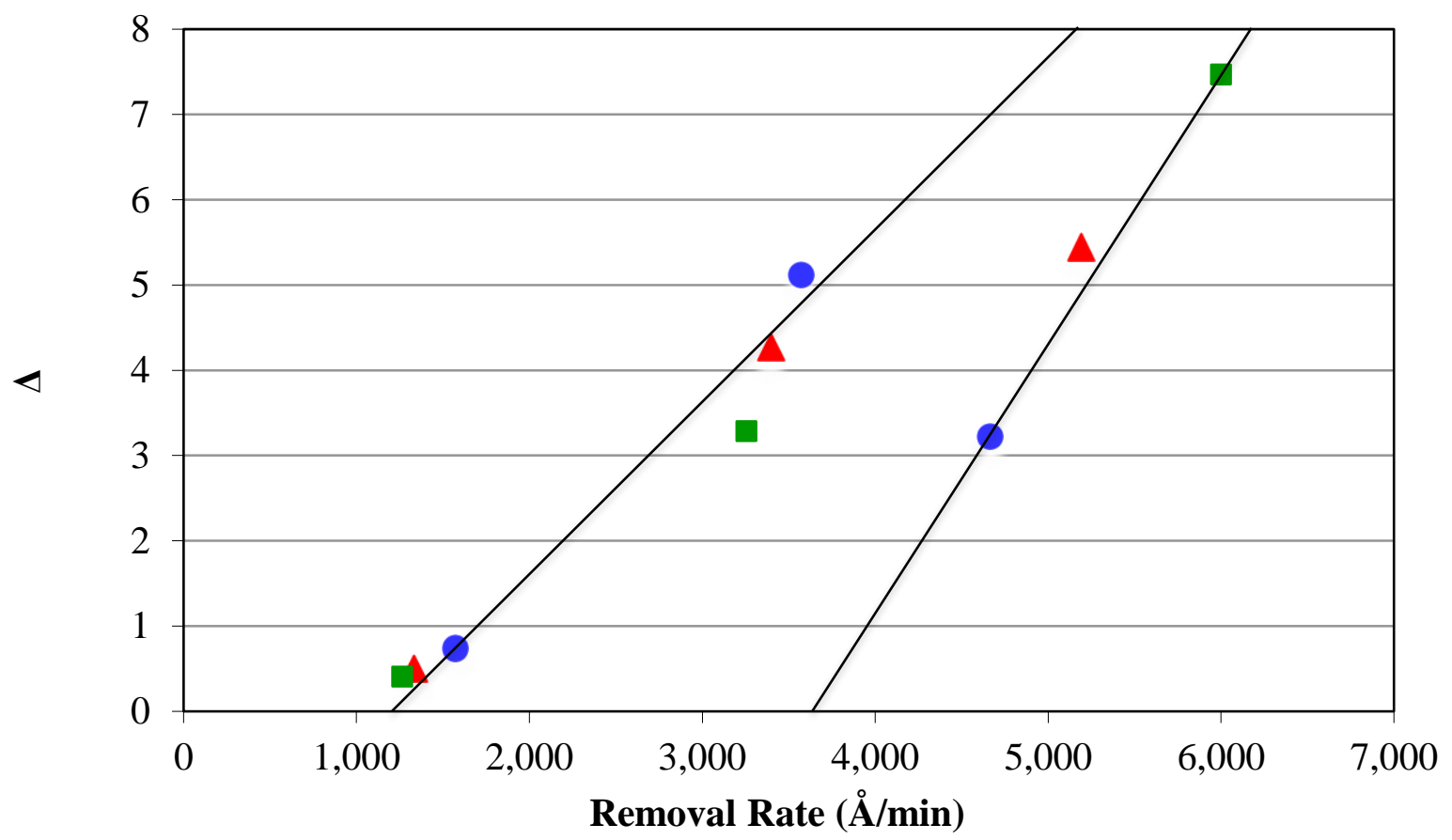

Figure 10: Copper Removal Rate vs $\Delta$ at sliding velocities of 1.2 (blue circles), 1.5 (red triangles) and 1.8 (green squares) $\mathrm{m} / \mathrm{s}$ - Using the Shinhan CCH23 conditioner. 